\title{
Quadrupole interaction of the $\mathrm{SrTiO}_{3}: \mathrm{V}^{4+}$ system
}

\author{
Th.W. Kool \\ University of Amsterdam
}

\begin{abstract}
Analysis were made to calculate the quadrupole interaction of the $\mathrm{Jahn}$-Teller $\mathrm{SrTiO}_{3}$ system from enhanced EPR spectra due to an applied static electric field. The calculations gave a value for the quadrupole constant$$
|P|=(-2.1420 .005) \times 10^{-4} \mathrm{~cm}^{-1} .
$$

EPR spectra of vanadium doped $\mathrm{SrTiO}_{3}$ crystals were reported before [1,2]. The spectra stemmed from a $\mathrm{V}^{4+}\left(d^{1}\right) S=1 / 2$ ion substituted for $\mathrm{Ti}^{4+}$. It was concluded that the $\mathrm{SrTiO}_{3}: \mathrm{V}^{+}$ impurity was a strong Jahn-Teller ion which resulted in a compression of the oxygen octahedron. Later, static electric field experiments in EPR confirmed this conclusion [2,3]. The static electric field gives rise to an appreciable lowering of the dielectric constant of $\mathrm{SrTiO}_{3}$, especially at low temperatures [4,5] leading to a better quality factor $Q$ of the resonant cavity. The quality factor $Q$ expresses the ratio between its energy loss per period [6]. Therefore this leads to a much better sensitivity of the EPR measurements and this resulted in observing small satellite lines stemming from 'forbidden' quadrupole transitions (see figure). Therefore we were able to calculate the quadrupole constant.

As said before in the electric field experiments we observe in EPR not only an enhancement of the EPR lines, which stem from centres with tetragonal axes parallel to the applied electric field, but also new small lines in the [100] direction (see figure).

The quadrupole term for the spin Hamiltonian is equal to (see also reference [7]):

$\mathcal{H}=\boldsymbol{I} . \boldsymbol{P} . \boldsymbol{I}$,

where $\boldsymbol{P}$ is the quadrupole coupling tensor.

In the principal axis system Eq.1 can be written as:

$\mathcal{H}=P_{x x} I_{x}^{2}+P_{y y} I_{y}^{2}+P_{z z} I_{z}^{2}$

In the case of axial symmetry the quadrupole coupling tensor is traceless so the Hamiltonian becomes:

$\mathcal{H}=P\left\{I_{z}^{2}-\frac{1}{3} I(I+1)\right\}$

$P=\frac{3}{2} P_{Z Z}$ 

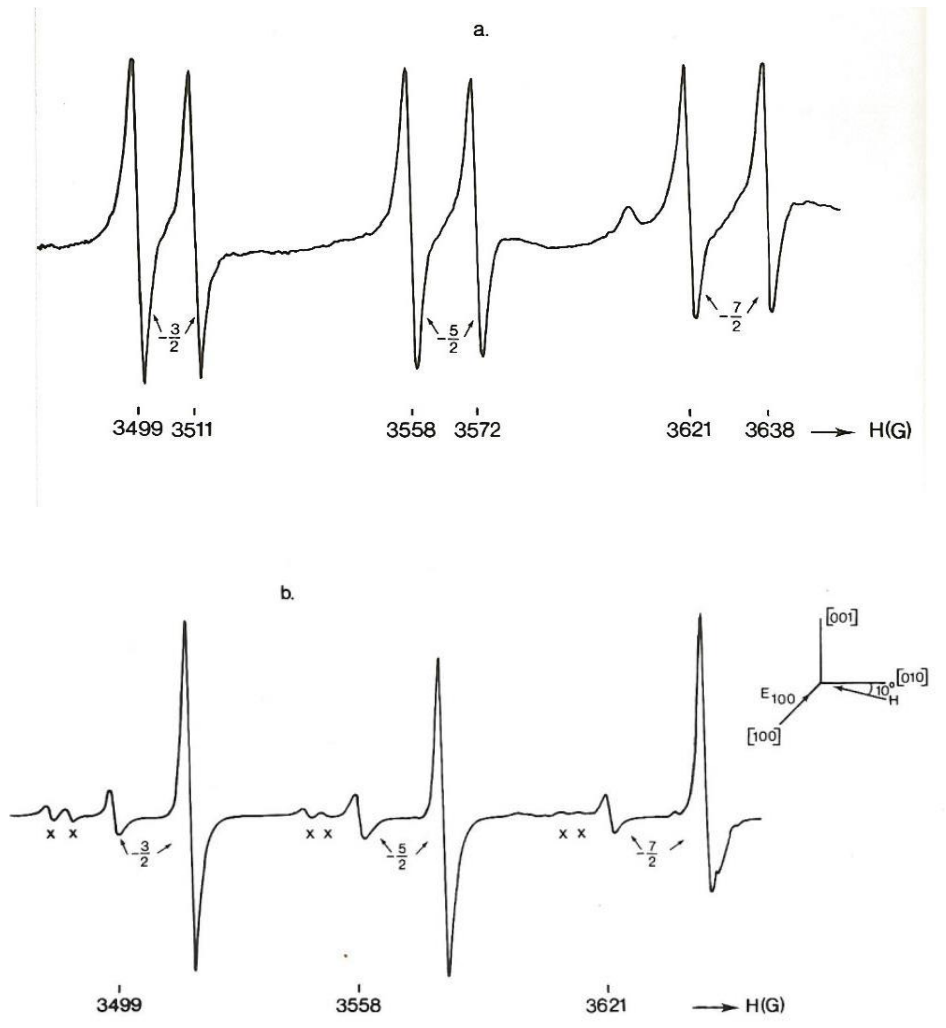

Figure. (a) EPR spectrum of the $\mathrm{SrTiO}_{3}: \mathrm{V}^{4+}$ system for $\boldsymbol{H}$ in the (001) plane, about $10^{\circ}$ away from the [010] axis, $\mathrm{T}=4.2 \mathrm{~K}$. (b) The same spectrum, but with an applied field $\boldsymbol{E} \|[100], E=21 \mathrm{kV} \mathrm{cm}^{-1}$. The lines marked $\times$ are 'forbidden' quadrupole transitions. (figure taken from references [3] and [2].

Assuming that the principal axis of the quadrupole coupling tensor coincide with those of the $g$ and hyperfine tensors we obtain:

$\mathcal{H}=\frac{P}{2}\left\{\left(\frac{3 A_{\|}^{2} g_{\|}^{2}}{g^{2} A^{2}}\right) \cos ^{2} \theta-1\right\}\left\{I_{Z}^{2}-\frac{1}{3} I(I+1\}-\frac{P}{2}\left(\frac{A_{\|} A_{\perp} g_{\|} g_{\perp}}{g^{2} A^{2}}\right) \sin 2 \theta\left\{I_{z} I_{x}+I_{x} I_{Z}\right\}+\right.$ $+\frac{P}{4}\left(\frac{A_{\perp}^{2} g_{\perp}^{2}}{g^{2} A^{2}}\right) \sin ^{2} \theta\left\{I_{x}^{2}-I_{y}^{2}\right\}$

where $g A=\left(A_{\|}^{2} g_{\|}^{2} \cos ^{2} \theta+A_{\perp}^{2} g_{\perp}^{2} \sin ^{2} \theta\right)^{1 / 2}$.

The first term gives a first order contribution to the energy of the state $\left|M_{S} M_{I}\right\rangle$ and shifts the energy states connected by $\Delta M_{I}=0$ equally. The second and third term mix respectively states $\left|M_{S}, M_{I} \pm 1\right\rangle$ and $\left|M_{S}, M_{I} \pm 2\right\rangle$ with $\left|M_{S}, M_{I}\right\rangle$. Because these terms are mixed, transitions of $\Delta M_{I}= \pm 1$ and $\Delta M_{I}= \pm 2$ become weakly allowed. 
At $\theta=0^{\circ}$ the $\Delta M_{I}= \pm 1$ and $\Delta M_{I}= \pm 2$ transitions have zero intensity, while at $\theta=90^{\circ}$ the $\Delta M_{I}$ $= \pm 1$ lines have zero intensity and the $\Delta M_{I}= \pm 2$ lines have intensity. In the $\mathrm{V}^{4+}: \mathrm{SrTiO}_{3}$ system we expect 12 possible transitions.

The energy differences for these $\Delta M_{I}= \pm 2$ transitions are:

$E\left(+\frac{1}{2}, M_{I} \pm 1\right)-\left(-\frac{1}{2}, M_{I} \mp 1\right)=h v=g \mu_{B}+A M_{I} \pm 4 P_{\theta} M_{I} \mp 2 G_{I}$

where $P_{\theta}=\frac{P}{2}\left\{\left(\frac{3 A_{\|}^{2} g_{\|}^{2}}{g^{2} A^{2}}\right) \cos ^{2} \theta-1\right\}$ and $G_{I}=\frac{g_{N}^{(I)} \mu_{B}^{(I)} H}{g A}\left(A_{\|}^{2} g_{\|}^{2} \cos ^{2} \theta+A_{\perp}^{2} g_{\perp}^{2} \sin ^{2} \theta\right)$

At $\theta=90^{\circ}, P_{\theta}$ in Eq. 5 becomes $-\frac{P}{2}$

From the position of the small quadrupole lines at $\theta=90^{\circ}$ (with the magnetic field $\vec{H}$ parallel to the [100] direction) and with the help of Eq. 5, we calculated that $|P|=(-2.142 \pm 0.005) \times 10^{-4} \mathrm{~cm}^{-1}$.

This value is in the same order of magnitude as those values found for $\mathrm{V}^{4+}$ in $\mathrm{GeO}_{2}$ [8] and $\mathrm{TiO}_{2}[9]$, respectively.

One very important assumption was made: There is no change in $|P|$ by changing the magnitude of the electric field. Because there was no change in hyperfine interaction on changing the magnitude of the electric field, we think that our assumption is right.

\section{References}

[1] Th.W. Kool and M. Glasbeek, Solid State Communications 32, 1099 (1979)

[2] K. Alex Müller and Tom W Kool, Properties of perovskites and other oxides, World Scientific (2010)

[3] Th.W. Kool and M. Glasbeek, J. Phys.: Condens. Matter 3, 9747 (1991)

[4] E. Sawaguchi, A. Kikuchi and Y. Kodera, J. Phys. Soc. Japan 17, 1666 (1962)

[5] E. Hegenbarth, phys. stat. sol. 6, 333 (1964)

[6] C.P. Poole Jr: In Electron Spin Resonance. A comprehensive treatise on experimental Techniques 2nd Ed. (Wiley, New York 1983)

[7] N.M. Atherton, Electron Spin Resonance. (Ellis Horwood Series in Phys. Chem.) 1973

[8] D.P. Madacsi, R.H. Bartram and O.R. Gilliam, Phys.Rev. B 7, 1817 (1973)

[9] H.J. Gerritsen and H.R. Lewis, Phys.Rev. 119, 1010 (1960) 